Reprod. Nutr. Dévelop., 1980, 20 (5 A), 1401-1414.

\title{
Digestion des glucides de la levure d'alcanes par le veau préruminant
}

\author{
par J. M. BESLE, Bernadette LASSALAS, P. THIVEND \\ Laboratoire de la Digestion des Ruminanis, I.N.R.A., \\ Theix, 63110 Beoumont France.
}

Summary. Digestion of alkane yeast carbohydrates by the preruminant calf.

Four 15-day old preruminant calves were fitfed with two single cannulae, one in the lower ileum and the other in the upper colon. They were fed (on dry-matter basis) a milk substitute containing 35 p. 100 of dried, defatted, micronized alkane yeast (Candida tropicalis). Yeast carbohydrate digestion was studied in the ileum, colon and feces of two calves at 48, 72 and 102 days of age (three periods), using 1 p. 100 of chromic oxide in the diet as a marker. During each period, samples were collected every 2 hours and then pooled to obtain a sample from each part of the digestive tract.

The carbohydrates were separated into $40^{\circ} \mathrm{C}$ water-soluble (neutral and non-neutral) and water-insoluble fractions (fig. 1), and the composition of each was determined by hydrolysis and ion-exchange chromatography.

The alkane yeast contained almost 22 p. 100 (in DM) of carbohydrate (table 1), mainly composed of 7.5 p. 100 of non-soluble mannans and 10.1 p. 100 of glucans, both from the hull, and 0.3 p. 100 of soluble galactans.

The total carbohydrate content (table 2) was high in the ileum (23 to 28 p. 100 of DM) and low in the feces ( 3 to 8 p. 100 of DM). At the end of the small intestine, the soluble fraction contained some mannans and galactans partly bonded with non-carbohydrate compounds, but mainly neutral glucans (fig. 2).

The apparent digestibilities (table 3, fig. 3) varied between animals and increased with age (total yeast carbohydrates : calf : 0.53 to 0.75 ; calf $2: 0.62$ to 0.85 ). Mannans were better digested than glucans (calf $2: 0.95$ and 0.57 , respectively, at 102 days) ; the galactans were completely digested.

\section{Introduction.}

Parmi les différents produits susceptibles d'être incorporés dans les aliments d'allaitement du veau préruminant, les levures d'alcanes semblent être une source de protéines convenablement équilibrée en acides aminés indispensables, bien qu'elles soient légèremenł déficientes en acides aminés soufrés (Senez, 1973 ; Van Weerden et al., 1972) et peut-être aussi en histidine (Patureau-Mirand et al., 1974). L'utilisation digestive de ces protéines par le veau préruminant est satisfaisante (Paruelle et al., 1972 ; Van Weerden, 1972 ; Kirchgessner et Roth, 1973) bien que moins élevée que celle des protéines du lait. 
Les glucides des levures pourraient être en partie à l'origine de cette différence. Ils représentent environ 22 p. 100 de la matière sèche de la levure d'alcanes et sont constitués d'une faible fraction de glucides cytoplasmiques (tréhalose, « glycogène ») et d'une importante fraction de glucides pariétaux. Les études sur la structure de la paroi (Lampen, 1968 ; Korn et Northcote, 1960 ; Mac William, 1970) ont montré qu'elle contient des mannanes en position externe, liés à des diesters phosphoriques et à des peptides (Neumann et Lampen, 1966 ; Sentandreu et Northcote, 1968 ; Ballou et Raschke, 1974) et des glucanes en position interne. La digestion de ces glucides a été peu étudiée; seul le « glycogène » pourrait en principe être digéré par le veau. Les travaux de Carillo et Bocourt (1971) permettent de penser qu'ils ne sont pas attaqués par les enzymes digestives du porc. En revanche, Gaillard et Van Weerden (1976) ont montré qu'une partie d'entre eux peut être dégradée dans l'intestin grêle et surtout dans le gros intestin du veau préruminant.

Pour obtenir davantage d'informations à ce sujet, nous avons étudié chez cet animal, la digestion de la fraction glucidique des levures d'alcanes, à différents niveaux du tube digestif (iléon distal, côlon proximal, fèces) et à différents âges. Nous avons étudié la dégradation des principaux glucides solubles et insolubles dans l'eau et la formation des produits terminaux de la digestion microbienne.

\section{Matériel ef méthodes.}

Animaux ef régimes.

Quatre veaux mâles de race Frisonne ont été munis de deux canules simples, l'une à la fin de l'iléon et l'autre au début du côlon à l'âge d'environ deux semaines. Ils ont reçu avant l'opération, et pendant la semaine suivante, un aliment d'allaitement classique. Puis, durant une période de transition de 10 jours, ils ont été habitués progressivement à recevoir l'aliment expérimental. Celui-ci a été distribué en fonction du poids métabolique des veaux $\left(\mathrm{p}^{0,75}\right)$ à raison de $150 \mathrm{~g}$ d'aliment par $\mathrm{kg}$ de lait, au seau en deux repas par jour. Il contenait 35 p. 100 (de la matière sèche) de levure (Candida Tropicalis $\left(^{1}\right)$ ) micronisée (apportant 81,3 p. 100 des matières azotées totales et 20,7 p. 100 des glucides), 61 p. 100 de poudre de lactosérum réengraissé avec du suif (36 p. 100) et 2 p. 100 de complément minéral et vitaminique. Nous y avons incorporé en outre 1 p. 100 d'oxyde de chrome et 1 p. 100 de polyéthylène glycol (PEG), marqueurs de transit qui permettent de déterminer quantitativement la digestion des différents constituants du régime. Celui-ci contenait 31,8 p. 100 de matières azotées 36,5 p. 100 de glucides (28,9 p. 100 de lactose et 7,5 p. 100 de glucides de la levure), 22,3 p. 100 de lipides et 9,5 p. 100 de matières minérales par rapport à la matière sèche Le maintien en suspension de cel aliment a été satisfaisant.

Mesures ef prélèvements.

Les prélèvements ont été effectués sur 3 animaux. A l'âge moyen de 48, 72 ef 102 jours (périodes $1,2,3$ ) nous avons prélevé pendant 24 h consécutives, à intervalles de $2 \mathrm{~h}$, du contenu digestif dans l'iléon, le côlon et le rectum, de façon à constituer un

(1) Toprina LS, Société Française des Pétroles B.P. 10, avenue Paul-Doumer, 92401 Courbevoie. 
échantilion moyen par niveau. Les échantillons étaient traités dès l'émission du contenu, le laps d'attente ne dépassant pas $30 \mathrm{~min}$. Une partie (environ 25 p. 100) destinée à l'analyse des glucides, était congelée immédiatement à $-20^{\circ} \mathrm{C}$ puis lyophilisée et broyée. L'autre partie servait d'abord à la mesure du $\mathrm{pH}$ puis était congelée dans un flacon avant d'être utilisée pour les autres analyses, soit à l'état frais, soił après lyophilisation et broyage.

\section{Méthodes d'analyses.}

La teneur en matière sèche a été déterminée à l'étuve à $80^{\circ} \mathrm{C}$ par dessiccation des échantillons jusqu'à poids constant. L'ammoniaque a été dosée colorimétriquement par la méthode de Berthelot, modifiée par Weatherburn (1967) et automatisée par Michel (1971). Les acides gras volatils ont été analysés par chromatographie en phase gazeuse (Rigaud et Journet, 1970). L'oxyde de chrome a été tout d'abord oxydé en $\mathrm{CrVI}+$ par la méthode de Bolin, King et Klosterman (1952) modifiée (l'oxydation se fait par le mélange nitro perchlorique au lieu du mélange sulfo perchlorique), puis dosé colorimétriquement suivant le procédé automatisé de Mathieson (1970). Le polyéthylène glycol a été déterminé par la méthode de Malawer et Don Powell (1967) modifiée (lecture à $420 \mathrm{~nm}$, temps de stabilisation de $1 \mathrm{~h} \mathrm{15)}$ ).

Extraction aqueuse ef purification des glucides. - Le schéma de fractiónnement glucidique est indiqué à la figure 1 . Nous avons utilisé comme agent d'extraction l'eau

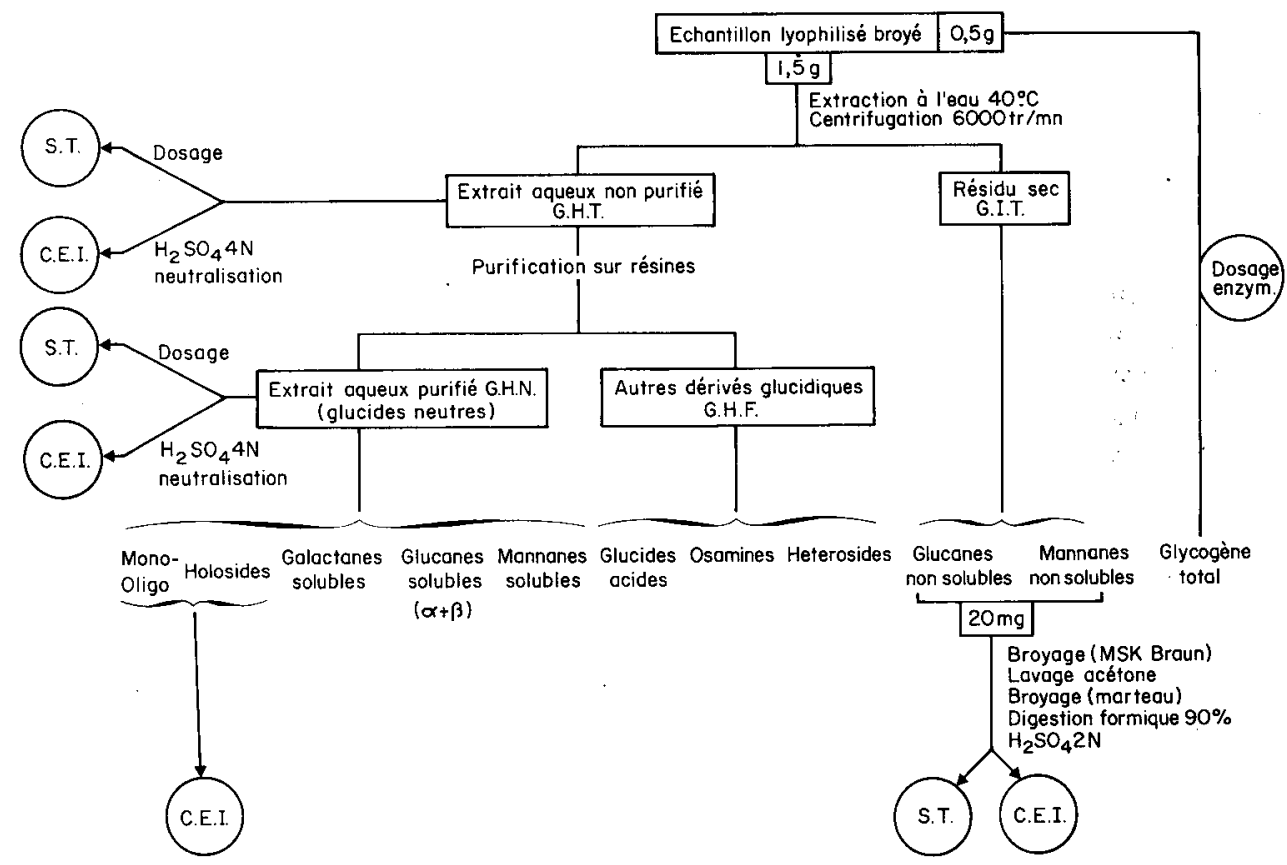

FIG. 1. - Fractionnement glucidique. C.E.I. = analyse par chromatographie échangeuse d'ions ; S.T. = dosage des sucres totaux ; G.H.T. = glucides hydrosolubles totaux ; G.H.F. = glucides hydrosolubles fixés pàr purification ; G.H.N. = glucides hydrosolubles neutres ; G.I.T. = glucides insolubles totaux. 
distillée additionnée de 1 p. 1000 de chlorure mercurique pour inhiber toute dégradation d'origine microbienne ou enzymatique. Nous avons procédé de la façon suivante : environ $1,5 \mathrm{~g}$ d'échantillon broyé est pesé exactement dans un pot de centrifugeuse de $500 \mathrm{ml}$ taré puis traité successivement, pendant deux fois une heure puis deux fois $30 \mathrm{~min}$, avec $40 \mathrm{ml}$ de solution mercurique pour les trois premières extractions et $25 \mathrm{ml}$ pour la dernière dans un bain marie à agitation à $40^{\circ} \mathrm{C}$. On centrifuge chaque fois à $6000 \mathrm{tr} / \mathrm{min}$ et on recueille le surnageant dans une fiole de $150 \mathrm{ml}$ que l'on ajuste en fin d'opération. Le résidu est séché dans le pot de centrifugation à l'étuve à $80^{\circ} \mathrm{C}$ pendant $48 \mathrm{~h}$ puis il est pesé, récupéré et broyé au broyeur à marteau.

L'extrait aqueux brut obtenu contient la tolalité des glucides hydrosolubles à $40^{\circ} \mathrm{C}$ (GHT = glucides hydrosolubles totaux). Il est purifié sur résines par la méthode de Montreuil (1957) modifiée (Besle et Pitiot, 1976), afin de faire la part des glucides neutres (GHN) el des autres composés glucidiques fixés lors de la purification (GHF = hétérosides + osamines + acides uroniques).

Fractionnement ef dosage des glucides solubles. - Les sucres totaux des GHT et des GHN sont évalués globalement par la méthode à l'orcinol sulfurique de Bial (1902) aułomatisée (Besle, 1974), à laquelle nous avons apporté diverses modifications, afin d'augmenter la sensibilité et la rapidité :

- la bobine de mélange orcinol - acide sulfurique est plongée dans le bain-marie à $95^{\circ}$;

- la bobine de refroidissement en sortie de bain-marie est supprimée ; dans ce cas, le coefficient de variation pour 20 dosages d'une solution de saccharose à $10 \mu \mathrm{g} / \mathrm{ml}$ n'est pas accru ;

- la coloration est mesurée à $510 \mathrm{~nm}$ afin d'éliminer l'interférence éventuelle des protéines dans le cas du dosage des extraits aqueux non purifiés ;

- la bulle qui s'intercale entre l'échantillon ef le liquide de rinçage est éliminée par un cactus-débulleur avant dosage car elle se substitue au liquide de prélèvement ef, en ne diluant pas le réactif sulfurique, provoque une réponse parasite du colorimètre.

Le temps de prélèvement est d'une minute, celui du rinçage de deux minutes.

Les mono, di et triholosides des extraits aqueux déféqués $(-18 \mathrm{ml}$ d'extrait $+1 \mathrm{ml}$ d'acétate de zinc 30 p. $100 \mathrm{p} / \mathrm{v}+1 \mathrm{ml}$ de ferrocyanure de potassium 15 p. $100 \mathrm{p} / \mathrm{v}$ ) sont séparés par chromatographie échangeuse d'ions (Besle, 1974).

Les polyholosides hydrosolubles ont été hydrolysés par $\mathrm{H}_{2} \mathrm{SO}_{4} 4 \mathrm{~N}$ pendant $4 \mathrm{~h}$ à $120^{\circ} \mathrm{C}$ au bain d'huile à reflux. Dans ces conditions, déterminées par une cinétique d'hydrolyse, le mannose, le glucose et le galactose ne sont pas détruits ; par contre 54 p. 100 du ribose ont disparu. Ce taux de dégradation, identique pour deux essais nous permet d'estimer la concentration avant hydrolyse.

Une fraction de l'hydrolysat est ensuite analysée par chromatographie échangeuse d'ions après avoir été au préalable tamponnée par une solution de borate à $\mathrm{pH} 8\left(\mathrm{H}_{3} \mathrm{BO}_{3}, 0,1 \mathrm{M}-\mathrm{NaOH}\right)$ ef neutralisée par de la soude $4 \mathrm{~N}$.

Analyse des glucides du résidu de l'extraction aqueuse. - Le résidu de l'extraction aqueuse contient les glucides de la paroi de la levure. La structure de celle-ci est détruite par broyage avec des microbilles de verre (Bonaly et al., 1971).

Après lavage à l'acétone et séchage, le résidu obtenu esł broyé au broyeur à marteau pendant $4 \mathrm{~min}$, puis il est hydrolysé suivant la méthode de Bonaly et al. 
(1971) modifiée par Caudy (1974) : la digestion à l'acide formique 90 p. 100 est suivie d'une hydrolyse par $\mathrm{H}_{2} \mathrm{SO}_{4} 2 \mathrm{~N}$ pendant $2 \mathrm{~h}$ à $120^{\circ} \mathrm{C}$ sous reflux. Une cinétique d'hydrolyse nous a permis de vérifier la validité de ces conditions opératoires. Le ribose esł conservé à 91 p. 100. L'hydrolysat est ensuite neutralisé comme précédemment et chromatographié après dosage préalable des sucres tołaux (GIT).

Dosage du glycogène. - Le glycogène a été dosé sur l'échantillon initial broyé avec un appareil MSK Braun ( ${ }^{2}$ ), puis au broyeur à marteau, suivant la méthode enzymatique de Thivend, Mercier et Guilbot (1972), proposée pour l'amidon mais qui peut être utilisée également pour le dosage du glycogène.

\section{Résultats.}

Les animaux ont toujours bien consommé l'aliment pendant toute la durée de l'expérience, et leur état sanitaire a été satisfaisant. En particulier, ils n'ont pas eu de diarrhée. La teneur en matière sèche des fèces a été voisine de 15 p. 100 au cours des six premières semaines ef plus élevée ultérieurement (de 21 à 30 p. 100).

La croissance a été modeste : de 500 à $833 \mathrm{~g} / \mathrm{j}$ à la première période et de $400 \mathrm{à}$ $560 \mathrm{~g} / \mathrm{j}$ à la troisième. Ceci est dô essentiellement à la nature du régime étudié qui était beaucoup plus riche en levures que ceux utilisés par ailleurs, mais aussi aux manipulations effectuées sur les animaux (prélèvements) et à la présence de canules intestinales.

Composition glucidique de la levure d'alcanes (tabl. 1).

TABLEAU 1

Composition glucidique de la levure d'alcanes en p. 100 de la mafière sèche

\begin{tabular}{|c|c|c|c|}
\hline Glucides & $\begin{array}{l}\text { Solubles } \\
\text { dans l'eau }\end{array}$ & $\begin{array}{l}\text { Insolubles } \\
\text { dans l'eau }\end{array}$ & Totaux \\
\hline 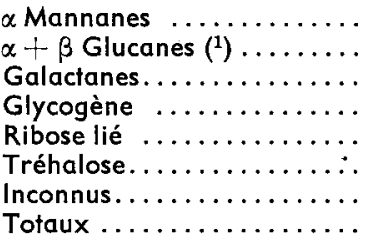 & $\begin{array}{l}1,66 \\
0,31 \\
0,28 \\
\overline{0,41} \\
0,02 \\
0,02 \\
2,70\end{array}$ & $\begin{array}{c}7,51 \\
10,14 \\
- \\
1,10 \\
1,20 \\
- \\
\overline{18,85}\end{array}$ & $\begin{array}{r}9,17 \\
10,45 \\
0,28 \\
1,10 \\
1,61 \\
0,02 \\
0,02 \\
21,55\end{array}$ \\
\hline
\end{tabular}

(1) Le glycogène est compris dans la fraction « $\alpha+\beta$ glucanes ».

La levure d'alcanes contient 2,7 p. 100 de glucides hydrosolubles (tréhalose, ribose, galactanes, mannanes, glucanes) et près de 20 p. 100 de glucides insolubles dans l'eau dónt 7,5 p. 100 de mannanes et 10,1 p. 100 de glucanes. Ces deux derniers résultats sont en accord avec ceux de Nabeshima, Tanaka ef Fukui (1970) et de Caudy

( ${ }^{2}$ ) Etablissements Roucaire 20, avenue de l'Europe, 78140 Velizy. 
(1974). Par contre, parmi les glucides solubles seuls le tréhalose et les mannanes ont été mis en évidence par d'autres auteurs (Eddy, Phil et Rudin, 1958, chez Saccharomyces cerevisiae) ; la présence de galactanes et de glucanes solubles est peut-être due au traitement subi par la levure (séchage, dégraissage, broyage).

Nous n'avons dosé que 1,1 p. 100 de glycogène ( $\alpha$ glucane) par méthode enzymatique alors que l'équipe japonaise en a trouvé 9,8 p. 100 par hydrolyse à la potasse (Trevelyan et Harrisson, 1956). La composition en glycogène dépend des souches, mais il est fort possible que nous l'ayons évaluée par défaut à cause de la structure de ce glucide qui est fortement associé à la paroi (Jeanloz, 1944 ; Northcote et Horne, 1952) et échappe probablement à l'action de l'amyloglucosidase. La fraction attaquable par l'enzyme permet tout de même d'apprécier la part pouvant être hydrolysée dans l'intestin grêle, qui esț très faible. De ce fait, nous n'avons pas différencié dans l'analyse les $\alpha$ ef $\beta$ glucanes.

Composition glucidique des contenus infestinaux (tabl. 2, fig. 2).

La digestion des glucides a été étudiée uniquement chez les deux animaux présentant les meilleurs résultats zootechniques. La teneur en glucides solubles ef insolubles rapportée à la matière sèche du contenu digestif diminue dans les parties distales de l'intestin et avec l'âge de l'animal. Qualitativement, elle est très comparable pour les 2 animaux étudiés. Le lactose a pratiquement disparu à la fin de l'intestin grêle. Dans quelques rares cas, il subsiste en très faibles quantités (maximum 0,06 p. 100 de la matière sèche). Les mannanes se répartissent en une fraction insoluble et une fraction soluble importante. Celle-ci contient des mannanes neutres et une grande part de mannohétérosides (fig. 2). Les glucanes ont une répartition semblable mais ils sont presque entièrement neutres à l'état soluble. Tous les galactanes sont solubles, la

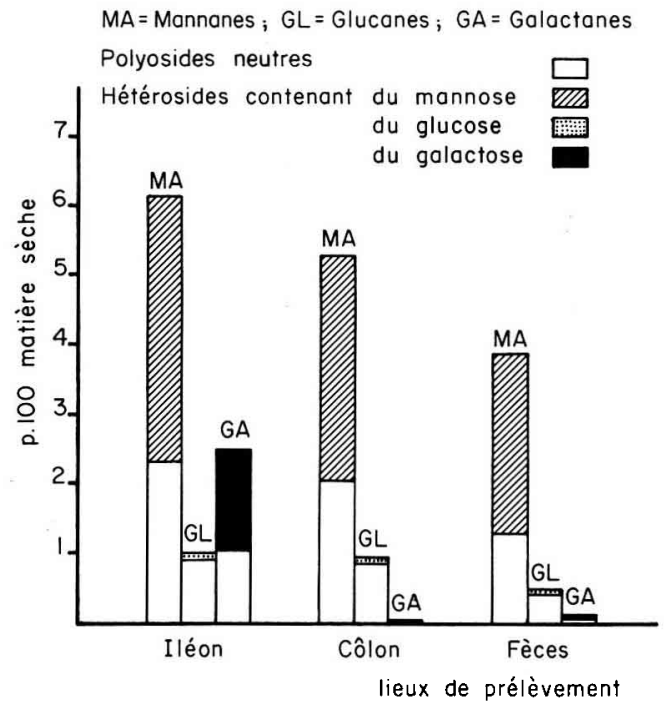

FIG. 2. - Composition en polyosides neutres ef en hétérosides des extraits aqueux (Veau $\mathrm{n}^{0} 1,48$ jours). 


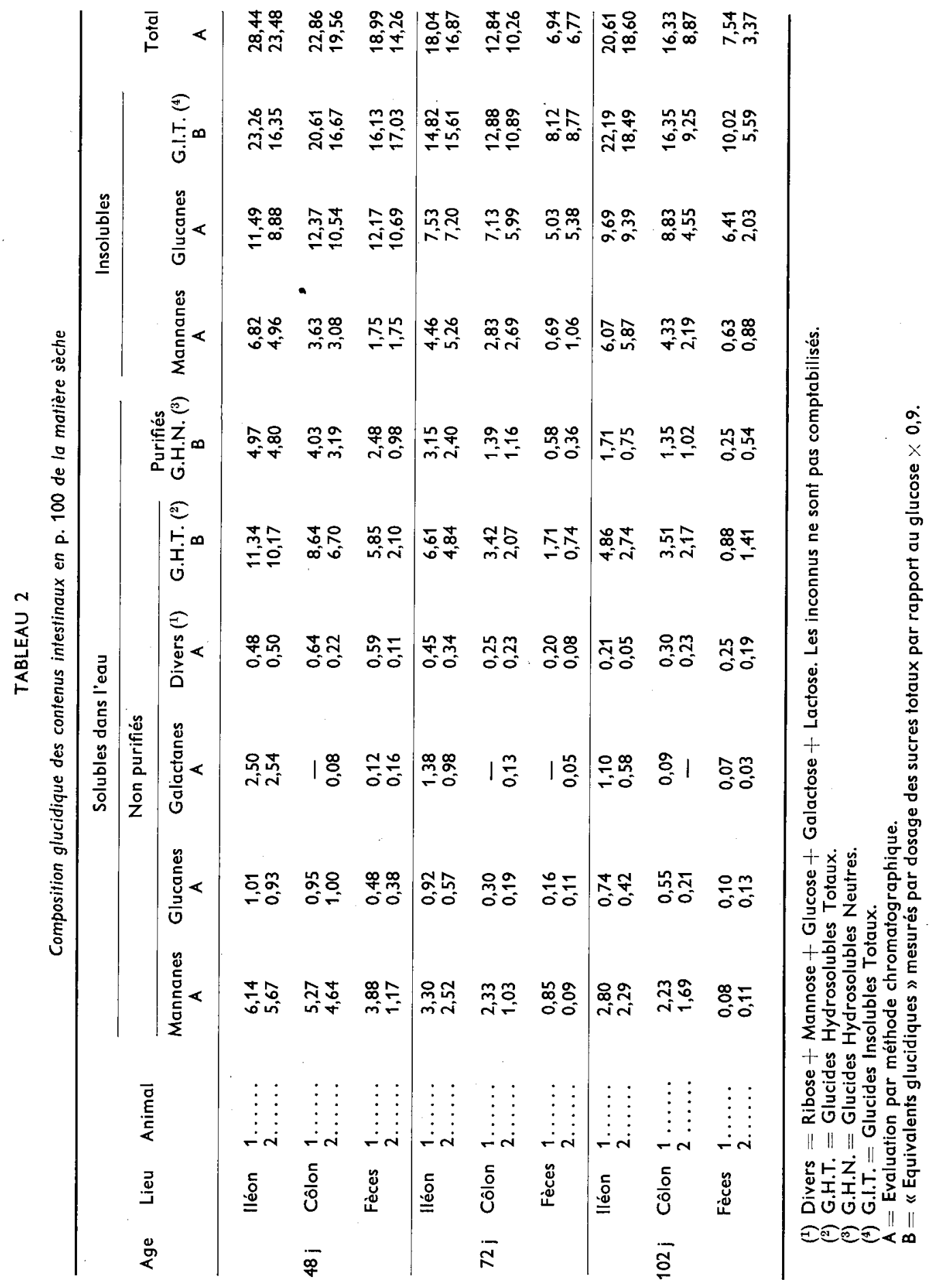


moitié seulement étant neutre dans l'iléon. lis ont déià presque complètement disparu dans le côlon. Le rapport «glucanes/mannanes 》 du résidu augmente tout au long du tube digestif (de 1,35 dans l'aliment à 10,0) dans les fèces. Le rapport « glucanes solubles/mannanes solubles » (valeurs extrêmes 0,28 et 0,12 ) varie peu, il est proche de celui trouvé par Cawley et Letters (1968) après digestion de la paroi de Saccharomyces cerevisiae par la pronase $(0,22)$.

Les glucides endogènes s'ajoutent aux glucides d'origine alimentaire mais nous n'avons pu les dissocier. L'importante fraction manno et galactohétérosidique faif penser qu'elle a partiellement une origine endogène.

D'une façon générale la concentration en oses neutres est faible : le mannose apparaît dans le caecum et se maintient à une faible concentration jusqu'à la fin du tube digestif (maximum 0,10 p. 100 de la matière sèche). Le glucose, déjà présent à la fin de l'iléon, augmente jusque dans le côlon (maximum 0,22 p. 100 de la matière sèche). La concentration en galactose libre suit celle des galactanes mais reste toujours faible (maximum 0,26 p. 100 de la matière sèche). Nous avons inventorié, principalement dans l'iléon, 10 pics correspondant à des glucides inconnus dans les extraits aqueux. Ce sont vraisemblablement des oligosides neutres (maximum 1,26 p. $100 \mathrm{MS}$ ) contenant du mannose, du glucose et du galactose. Leur concentration diminue avec l'âge.

Les GHN suivent une évolution parallèle aux GHF ( $r=0,949$ pour le veau 1, $r=0,986$ pour le veau 2), ils sont utilisés de la même manière. Pour cette raison, nous ne donnerons par la suite que les résultats d'analyse des GHT.

Imporfance et lieux de la digestion des glucides.

Pour quantifier la digestion nous n'avons utilisé que l'oxyde de chrome et non le PEG car la concentration de ce dernier dans les contenus nous a paru aberrante (elle a été parfois plus élevée dans les fèces que dans l'iléon). Ce phénomène, probablement dû à la méthode de dosage, serait lié à la nature des protéines dans les contenus (Smith, 1958).

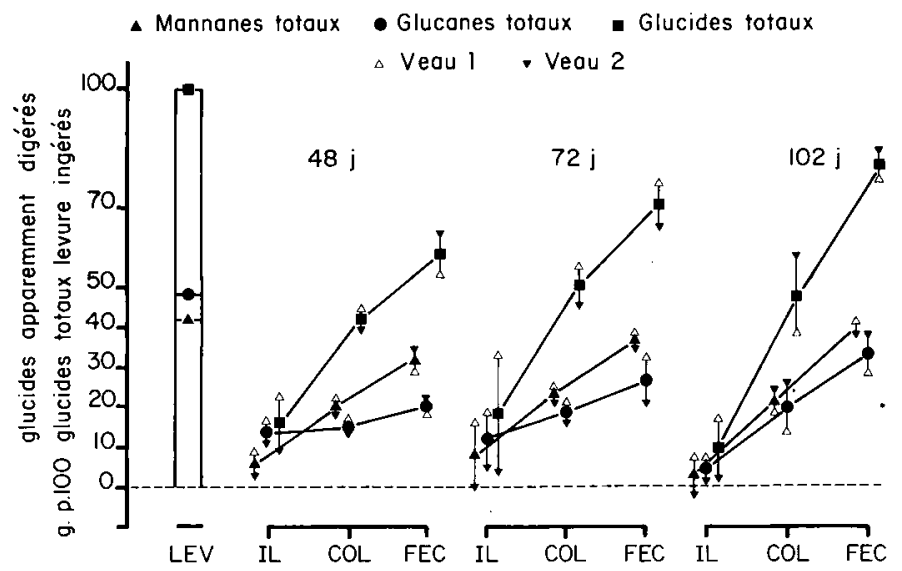

FIG. 3. - Quantités de glucides opparemment digérées le long du tube digestif ( $\mathrm{g} \mathrm{p} .100 \mathrm{~g}$ de la somme des glucides de levure ingérés). $\mathrm{LEV}=$ levure ; IL $=$ iléon $; \mathrm{COL}=$ côlon ; FEC $=$ fèces. 
La digestion des glucides a été étudiée sur trois fractions majeures : ensemble des glucides, glucanes, mannanes. Nous avons regroupé, pour ces deux derniers types, les fractions insolubles et solubles car nous estimons que ces dernières sont en grande partie des produits de la dégradation des polymères insolubles. Les quantités de glucides digérés augmentent à partir de la fin de l’intestin grêle (fig. 3). La digestibilité apparente des trois fractions (tabl. 3) varie beaucoup d'un animal à l'autre. Elle croît avec l'âge, passant respectivement chez les veaux 1 (et 2), de 0,53 à 0,76 $(0,62$ à 0,85$)$ pour l'ensemble des glucides, de 0,69 à $0,95(0,82$ à 0,90$)$ pour les mannanes, de 0,37 à $0,57(0,41$ à 0,79$)$ pour les glucanes. Les mannanes, mieux utilisés que les glucanes, sont presque entièrement digérés à l'âge de 102 jours.

TABLEAU 3

Digestion des principaux glucides de la levure à différents niveaux du tube digestif

\begin{tabular}{|c|c|c|c|c|c|c|c|}
\hline \multirow{2}{*}{$\begin{array}{l}\text { Fractions } \\
\text { étudiées }\end{array}$} & \multirow{2}{*}{$\frac{\text { Age (j) }}{\text { Animal }}$} & \multicolumn{2}{|c|}{48} & \multicolumn{2}{|c|}{72} & \multicolumn{2}{|c|}{102} \\
\hline & & 1 & 2 & 1 & 2 & 1 & 2 \\
\hline \multirow{3}{*}{$\begin{array}{c}\text { Glucides } \\
\text { totaux }\end{array}$} & \multirow{3}{*}{$\begin{array}{l}\text { Quantités ingérées }(\mathrm{g} / \mathrm{j}) \\
\text { Digestibilité apparente }{ }=100 \\
\text { Quantités digérées dans l'intestin } \\
\text { grêle (p. } 100 \text { du digestible) } \\
\text { Quantités digérées dans le gros } \\
\text { intestin (p. } 100 \mathrm{du} \text { digestible) }\end{array}$} & $\begin{array}{r}111,6 \\
52,7\end{array}$ & $\begin{array}{l}97,0 \\
62,4\end{array}$ & $\begin{array}{r}152,0 \\
76,7\end{array}$ & $\begin{array}{c}120,60 \\
65,7\end{array}$ & $\begin{array}{r}173,1 \\
76,3\end{array}$ & $\begin{array}{r}126,3 \\
84,7\end{array}$ \\
\hline & & 45 & 13 & 42 & 5 & 21 & 3 \\
\hline & & 55 & 87 & 58 & 95 & 79 & 97 \\
\hline \multirow{3}{*}{$\begin{array}{c}\text { Mannanes } \\
\text { totaux }\end{array}$} & \multirow{3}{*}{$\begin{array}{l}\text { Quantités ingérées }(\mathrm{g} / \mathrm{j}) \\
\text { Digestibilité apparente } \times 100 \\
\text { Quantités digérées dans l'intestin } \\
\text { grêle (p. } 100 \text { du digestible) } \\
\text { Quantités digérées dans le gros } \\
\text { intestin (p. } 100 \text { du digestible) }\end{array}$} & $\begin{array}{l}47,5 \\
68,7\end{array}$ & $\begin{array}{l}41,2 \\
82,1\end{array}$ & $\begin{array}{l}64,6 \\
87,9\end{array}$ & $\begin{array}{l}51,3 \\
86,4\end{array}$ & $\begin{array}{l}73,6 \\
94,7\end{array}$ & $\begin{array}{l}53,7 \\
89,7\end{array}$ \\
\hline & & 30 & 9 & 40 & -3 & 17 & -9 \\
\hline & & 70 & 91 & 60 & 103 & 83 & 109 \\
\hline \multirow{3}{*}{$\begin{array}{l}\text { Glucanes } \\
\text { totaux }\end{array}$} & \multirow{3}{*}{$\begin{array}{l}\text { Quantités ingérées }(\mathrm{g} / \mathrm{j}) \\
\text { Digestibilité apparente } \% 100 \\
\text { Quantités digérées dans l'intestin } \\
\text { grêle (p. } 100 \text { du digestible) } \\
\text { Quantités digérées dans le gros } \\
\text { intestin (p. } 100 \text { du digestible) }\end{array}$} & $\begin{array}{l}54,1 \\
37,5\end{array}$ & $\begin{array}{l}47,0 \\
40,6\end{array}$ & $\begin{array}{l}73,6 \\
64,0\end{array}$ & $\begin{array}{l}58,4 \\
43,5\end{array}$ & $\begin{array}{l}76,8 \\
57,2\end{array}$ & $\begin{array}{l}61,2 \\
79,5\end{array}$ \\
\hline & & 86 & 595 & 58 & 22 & 23 & 1 \\
\hline & & 14 & 41 & 42 & 78 & 77 & 99 \\
\hline
\end{tabular}

Les glucides de la levure sont principalement digérés dans le gros intestin, la proportion augmentant avec l'âge (chez le veau $1: 55$ à 79 p. 100 du digestible pour l'ensemble, 70 à 83 p. 100 pour les mannanes, 14 à 77 p. 100 pour les glucanes) (tabl. 3). Corrélativement, la part digérée dans l'intestin grêle, qui ne représente au maximum que 45 p. 100 du digestible, diminue et l'on obtient même des valeurs négatives pour les mannanes.

S'il y a peu de mannanes et de glucanes totaux digérés dans l'iléon, on observe cependant à cet endroit un début d'attaque de ces fractions, caractérisée par l'apparition de polymères solubles présents en quantités supérieures à celles ingérées, surtout pour les mannanes (fig. 4). Il en est de même pour les galactanes mais la fraction supplémentaire mise en évidence diminue avec l'âge. Ils ont presque entièrement disparu dans le côlon. Si l'on considère l'évolution avec l'âge de la digestion des fractions solubles et insolubles des mannanes et des glucanes (fig. 5), on observe dans l'intestin 
grêle d'une part une diminution de la digestibilité apparente des insolubles et d'autre part une disparition progressive des solubles, la résultante de ces évolutions se traduisant par une baisse de la digestibilité apparente de l'ensemble de chaque fraction. Dans le gros intestin, on remarque une importante augmentation de la dégradation de la fraction insoluble, principalement pour les glucanes.

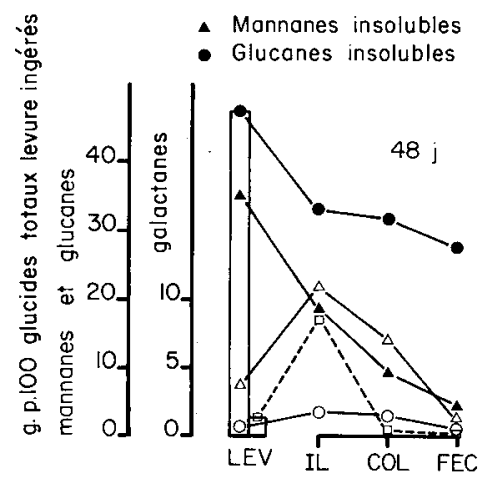

$\triangle$ Mannanes hydrosolubles
O Glucanes hydrosolubles Galactanes
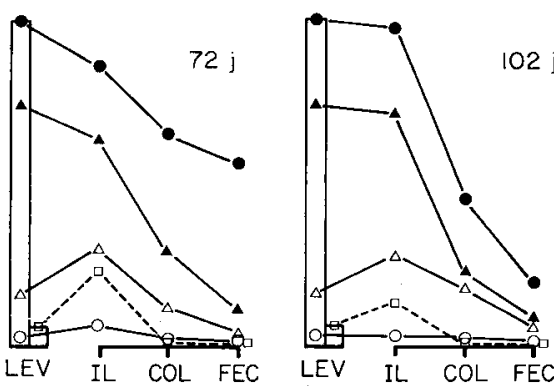

FIG. 4. - Evolution de la digestion des glucides dans le tube digestif (g p. $100 \mathrm{~g}$ de la somme des glucides levure ingérés, Veau $\mathrm{n}^{\circ} 2$ ).

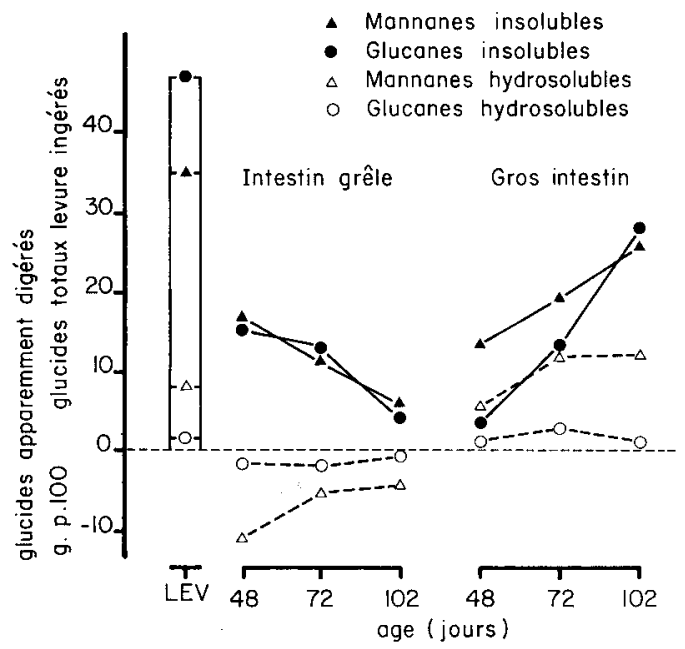

FIG. 5. - Evolution avec l'âge de la digestion des glucides

(g p. 100 de la somme des glucides de levure ingérés, Veau no 2 ). LEV $=$ levure.

\section{Produits terminaux de la fermentation microbienne.}

Dans l'iléon, la valeur du pH a été constante $(7,3)$, dans le côlon, elle a été plus faible et a varié de 6 à 6,4 , augmentant avec l'âge des animaux. Dans les fèces, nous avons observé, à l'âge de 48 jours, des valeurs comprises entre 6,6 et 6,8.

La concentration des acides gras volatils est faible dans l'iléon $(25,5 \pm 18,1$ mmoles/l); on y trouve presque exclusivement de l'acide acétique. Dans le côlon 
et les fèces, elle est plus élevée (respectivement 113,6 $\pm 26,1$ et 105,2 $\pm 39,6$ mmoles/l). Dans le côlon, le mélange est composé d'acide acétique, propionique et butyrique (en valeur relative respectivement $64 \pm 14 ; 22 \pm 8 ; 11 \pm 6$ p. 100 molaire). Dans les fèces les proportions sont semblables $(61 \pm 14 ; 26 \pm 14$; $10 \pm 4$ p. 100 molaire). Il n'y a pas d'évolution apparente avec l'âge des animaux.

La teneur en azote ammoniacal des contenus digestifś a varié de 22 à 61 mmoles par litre de contenu frais. Elle a eu tendance à diminuer dans l'iléon et dans le côlon avec l'âge des veaux.

\section{Discussion.}

Nos résultats montrent que les glucides de la levure d'alcanes peuvent être digérés par le veau préruminant. Déjà au niveau de l'iléon, nous avons mis en évidence une importante fraction polyosidique soluble. Il est vraisemblable que les mannanes solubles, en grande partie de nature hétérosidique, ne proviennent pas seulement des glucides endogènes mais aussi de la paroi de la levure. Il en est de même pour les galactanes, bien que, selon Roberts et Mac Farren (1953), ils puissent être aussi synthétisés dans l'intestin à partir du lactose. Les glucanes solubles sont principalement neutres, ils proviendraient surtout de la paroi interne de la levure. L'utilisation des glucides pariétaux semble donc se dérouler en trois stades:

Tout d'abord, ils sont solubilisés en polyosides plus courts. L'intestin grêle du veau ne possède aucune enzyme spécifique capable d'effectuer ces transformations. Les enzymes glycolytiques de la levure situés sur la paroi externe (Lampen,1968; Nurminen, Oura et Suomalainen, 1970) peuvent en être partiellement responsables, dans la mesure où elles ne sont pas détruites par le traitement (séchage, dégraissage, broyage). L'action d'enzymes d'origine microbienne (Nagasaki et al., 1966), de protéases diverses (Cawley et Letters, 1968 ; Mac William, 1970) y compris la pepsine et la trypsine (Eddy, Phil et Rudin, 1958) sur la paroi des levures, permettent d'obtenir des mannanes solubles liés à des peptides et à des diesters phosphoriques. Cette attaque se fait d'autant mieux que l'on ajoute des glycosidases au milieu (Caudy, 1974). Nous pensons donc que l'action conjuguée des glycosidases subsistantes de la levure, des protéases de l'intestin grêle et des enzymes de la microflore a pour résultat l'attaque d'une fraction appréciable de la paroi. D'après Carillo et Bocourt (1971), cette action n'est pas possible sur la levure vivante dans l'intestin du porc.

Dans un deuxième stade, les polymères solubles sont hydrolysés par la flore microbienne en glucose et en mannose. L'augmentation du rapport « glucanes/mannanes » du résidu insoluble ef la stabilité du rapport « glucanes solubles/mannanes solubles » montrent que les mannanes sont solubilisés puis utilisés plus rapidement que les glucanes.

L'élévation de la concentration en polyosides solubles dans l'iléon par rapport à celle de l'aliment pourrait laisser penser que, dans cette partie du tube digestif, le premier stade est plus rapide que le deuxième. Toutefois, cette hypothèse ne peut être retenue tant que nous ignorons la part des glucides endogènes du contenu intestinal. Par contre, la diminution de concentration des polyosides solubles dans le gros intestin nous permet de penser qu'à ce niveau le deuxième stade est plus rapide que le premier. 
Dans un troisième stade, les oses produits disparaissent rapidement, ils sont absorbés et (ou) utilisés par la flore microbienne. Dans l'intestin grêle l'absorption est active, celle du glucose est plus rapide que celle du mannose (Csaky et Ho, 1966, chez le rat ; Bihler, 1969, chez le hamster) ; dans le gros intestin, il est vraisemblable que ces oses sont surtout fermentés.

La baisse de digestibilité apparente de l'ensemble des glucides de la levure avec l'âge (fig. 5) dans l'intestin grêle pourrait être due, soit à une saturation des mécanismes de dégradation des glucides de la levure dans l'intestin grêle avec l'augmentation des quantités ingérées (surtout pour un taux élevé de glucides indigestibles), soif à une augmentation de la part de glucides endogènes insolubles, provenant notamment des mucus stomacaux et intestinaux (Snary et Allen, 1972).

L'augmentation avec l'âge de la part des glucides digérés dans le gros intestin traduit un accroissement de l'activité de la population microbienne dans cette partie du tube digestif ; toutefois, elle ne s'est pas manifestée par une augmentation de la concentration en acides gras volatils, puisque probablement, comme dans le rumen (Thorlacius et Lodge, 1973) leur absorption est accrue avec l'acidité du milieu.

Les résultats sur les produits terminaux de la fermentation se rapprochent dans l'iléon, de ceux d'Assan (1974) et de Chongo Garcia (1976) respectivement avec des régimes riches en amidon et en saccharose (mélasse). Dans le gros intestin, ils traduisent une activité fermentaire plus réduite que celle observée avec ces autres sources d'énergie.

Nos résultats doivent cependant être interprétés avec prudence. En effet, la digestibilité apparente mesurée est sans doute inférieure à celle obtenue sur l'animal non perfurbé, du fait des prélèvements qui accélèreraient le transit (Van Hellemond et Van Werden, 1973) ; elle est de plus sous-estimée si la récupération de l'oxyde de chrome est incomplète. Une autre cause d'erreur, difficile à apprécier pourrait provenir d'une éventuelle mauvaise représentativité de l'échantillon moyen collecté.

En conclusion, la digestion des glucides de la levure d'alcanes chez le veau préruminant a lieu surtout dans le gros intestin avec une grande variabilité selon l'animal, déjà signalée par Gaillard et Van Weerden (1976). Elle s'accroît avec l'âge (suite à l'adaptation de la flore microbienne) dans le gros intestin mais pas dans l'intestin grêle. Etant donné le taux élevé de levure incorporée, la digestibilité de l'aliment a été médiocre. Kaemmerer (1974) a observé également que le rat utilisaif mal les aliments à forte teneur en levure. Il est vraisemblable que, distribués en quantité plus faible, correspondant à une formule d'allaitement classique, les glucides seraient mieux utilisés.

La dégradation des glucides dans le gros intestin du jeune veau est un phénomène maintenant bien établi (Assan, 1974 pour les produits amylacés ; Chongo Garcia, 1976 pour le saccharose). Plus étonnante est celle d'une fraction non négligeable des glucides de la levure dans l'intestin grêle ou au niveau de l'iléon. Ce phénomène, déjà partiellement mis en évidence par Gaillard et Van Weerden (1976), semble complexe ef mettre en jeu l'action d'enzymes de la paroi de la levure, du tube digestif et de la microflore. Le mécanisme de cette digestion mériterait d'être approfondi. 
Remerciements. - A la Société Française des Pétroles BP, 10, quai Paul Doumer, 92401 Courbevoie, pour sa contribution financière. A Marie-Thérèse Beaufort, Michel Fabre, Marie-Paule Girard, Paulette Journaix, Jacques Lefaivre, Alain Vizet pour leur participation à la réalisation de ce travail.

\section{References}

ASSAN B. E., 1974. Contribution à l'étude de la digestion intestinale de l'amidon chez le veau préruminant. Th. Fac. Sci., Univ. Clermont-Ferrand.

BALLOU C. E., RASCHKE W. C., 1974. Polymorphism of the somatic antigen of yeast. Science, 184, 127-134.

BESLE J. M., 1974. Séparation des oses, des di et triholosides par chromatographie d'échange d'ions. Ann. Biol. anim. Bioch. Biophys., 14, 545-573.

BESLE J. M., PITIOT M., 1976. Extraction et purification des glucides : application à divers aliments dérivés du soja. Ann. Biol. anim. Bioch. Biophys., 16, 753-772.

BIAL M., 1902. Die Diagnose der Pentosurie. Dt. Med. Wschr., 28, 263-264.

BIHLER 1., 1969. Intesfinal sugar transport : ionic activation and chemical specificity. Biochim. biophys. Acto, 183, 169-181.

BOLIN D. W., KING R. P., KLOSTERMAN E. W., 1952. A simplified method for the determination of chromic oxide $\mathrm{Cr}_{2} \mathrm{O}_{3}$ when used as an index substance. Science, 116, 634-635.

BONALY R., MOULKY H., TOUIMI BENJELLOUN A., PIERFITTE M., 1971. Etude des parois de levures du genre rhodotorula. II. Influence des conditions de culture sur la composition chimique des parois. Biochim. biophys. Acto, 244, 484-494.

CARRILLO O., BOCOURT R., 1971. Influence of the cell wall on the digestibility of baker's yeast. Rev. cubana cienc. agric., 5, 341-346.

CAUDY P., 1974. Contribution à l'étude de la structure chimique de la paroi de la levure Candida Tropicalis. Th. Univ. Paris-Sud, Centre d'Orsay.

CAWLEY T. N., LETTERS R., 1968. Phosphoglycopeptides from yeast cell walls. Biochem. J., 110, 9 P-10 P.

CHONGO GARCIA B. M., 1976. Etude de la digestion intestinale de la mélasse de canne à sucre chez le veau préruminant. Th. Univ. Clermont II.

CSAKY T. Z., HO P. M., 1966. Active transport of D-Mannose in the small intestine. Life Sci., 5, 1025 1030.

EDDY A. A., PHIL D., RUDIN A. M., 1958. Part of the yeast surface apparently involved in floculation. J. Inst. Brew., 64, 19-21.

GAILLARD B. D. E., VAN WEERDEN E. J., 1976. The digestion of yeast cell wall polysaccharides in veal calves. Br. J. Nutr., 36, 471-478.

JEANLOZ R., 1944. Recherches sur l'amidon XXVIII. Le glycogène de levure natif. Helv. chim. Acia, 27, 1501-1509.

KAEMMERER K., 1974. Bedeutung und Verträglichkeit von Alkanhefen als Nahrungseiweiß. Züchfungskunde, 46, 56-72, 139-155.

KIRCHGESSNER M., ROTH F. X., 1973. Yeasts grown on n-paraffins for fattening calves. Züchfungskunde, 45, 208-214.

KORN E. D., NORTHCOTE D. H., 1960. Physical and chemical properties of polysaccharides and glycoproteins of the yeast cell-wall. Biochem. J., 75, 12-16.

LAMPEN J. O., 1968. External enzymes of yeast : their nature and formation. Antonie van Leenwenhoek, 34, 1-18.

Mac WILLIAM I. C., 1970. The structure, syntheses and fonctions of the yeast cell wall. A review. J. Inst. Brew., 76, 524-535.

MALAWER S. J., DON POWELL W., 1967. An improved turbidimetric analysis of polyethylene glycol utilizing an emulsifier. Gastroenterology, 53, 25C-256.

MATHIESON J., 1970. The automated estimation of chromic oxide. Proc. Nutr. Soc., 29, 30 A-31 A.

MICHEL M. C., 1971. Analyse quantitative de quelques substances azotées ef glucidiques en milieu biologique. Essai de rationalisation. Th. Fac. Sci., Univ. Clermont-Ferrand. 
MONTREUIL J., 1957. Glycoproteides. Bull. Soc. Chim. biol., 39, suppl. III, 1-92.

NABESHIMA S., TANAKA A., FUKUI S., 1970. Studies on the utilization of hydrocarbons by microorganisms. J. Ferment. Technol., 48, 556-562.

NAGASAKI S., NEUMANN N. P., ARNOW P., SCHNABLE L. D., LAMPEN J. O., 1966. An enzyme which degrades the walls of living yeast. Bioch. biophys. Res. comm., 25, 158-164.

NEUMANN N. P., LAMPEN J. O., 1966. Purification and properties of yeast invertase, Fed. Proc., 25, 588-589.

NORTHCOTE D. H., HORNE R. W., 1952. The chemical composition and structure of the yeast cell wall. Bioch. J., 51, 232-236.

NURMINEN T., OURA E., SUOMALAINEN H., 1970. The enzymic composition of the isolated cell wall and plasma membrane of baker's yeast. Biochem. J., 116, 61-69.

PARUELLE J. L., TOULLEC R., FRANTZEN J. R., MATHIEU C. M., 1972. Utilisation des protéines par le veau préruminant à l'engrais. I. - Utilisation digestive des protéines de soja et des levures d'alcanes incorporées dans les aliments d'allaitement. Ann. Zootech., 21, 319-331.

PATUREAU-MIRAND P., TOULLEC R., PARUELLE J. L., PRUGNAUD J., PION R., 1974. Influence de la nature des matières azotées des aliments d'allaitement sur l'aminoacidémie du veau préruminant. Ann. Zootech., 23, 343-358.

RIGAUD J., JOURNET M., 1970. Méthode de dosage des acides gras volatils dans le liquide du rumen. Ann. Biol. anim. Biochim. Biophys., 10, 151-157.

ROBERTS H. R., McFARREN E. F., 1953. The chromatographic observation of oligosaccharides formed during the lactase hydrolysis of lactose. J. Dairy Sci., 42, 620-632.

SENEZ J. C., 1973. Production industrielle de produits alimentaires à partir des hydrocarbures. Rec. Med. vét., 149, 889-902.

SENTANDREU R., NORTHCOTE D. H., 1968. The structure of a glycopeptide isolated from the yeast cell wall. Biochem. J., 109, 419-432.

SMITH R. H., 1958. Substances in the calf alimentary tract interfering in the determination of P. E. G. Nafure, 182, 260-261.

SNARY D., ALLEN A., 1972. Studies on gastric mucoproteins. The production of radioactive mucoproteins by pig gastric mucosa scrapings in vitro. Biochem. J., 127, 577-587.

THIVEND P., MERCIER C., GUILBOT A., 1972. Determination of starch with glucoamylase, 100-105. In Methods of carbohydrate chemistry, WHISTLER R. L., WOLFROM M. C., Acad. Press, New York and London.

THORLACIUS S. O., LODGE G. A., 1973. Absorption of steam volatile fatty acids from the rumen of the cow as influenced by diet, buffers and pH. Con. J. Anim. Sci., 53, 279-288.

TREVELYAN W. E., HARRISSON J. S., 1956. Studies on yeast metabolism. 7 - Yeast carbohydrate fractions. Separation from nucleic acid, analysis and behaviour during anaerobic fermentation. Biochem. J., 63, 23-33.

VAN HELLEMOND K. K., VAN WEERDEN E. J., 1973. Milk proteins substitutes in rations of veal calves. Proc. Nutr. Soc., 32, 231-235.

VAN WEERDEN E. J., 1972. Cité par SHACKLADY L. A. et GATUMEL E., dans Proteins from hydrocarbons, GOUNELLE de PONTANEL H., Aix-en-Provence, 27-52.

VAN WEERDEN E. J., SHACKLADY C. A., VAN DER WAL P., SCHUTTE J. B., 1972. Mełabolisable energy of yeast grown on pure n-paraffins and its use in rations for broiler chicks. Arch. Geflügelk., 1, 1-4.

WEATHERBURNN. W., 1967. Phenol hypochlorite reaction for determination of ammonia. Analyt. Chem., 39, 971-974. 